\title{
Bhabha scattering at very small angles at LEP
}

\author{
W. Beenakker* \\ CERN, Theory Division, CH-1211 Geneva 23, Switzerland \\ and \\ B. Pietrzyk \\ Laboratoire de Physique des Particules LAPP, IN2P3-CNRS, \\ F-74941 Annecy-le-Vieux Cedex, France
}

\begin{abstract}
We have reanalysed the various terms contributing to the luminosity calculation in the angular region of the new silicon-tungsten calorimeters at LEP. The calculations of the QED $\mathrm{O}(\alpha)$ corrections and of the higher-order leading $\log$ corrections to the $t$-channel photon-exchange contribution have a technical precision of $0.03 \%$, comparable to that shown for larger angles. The relative contribution of terms involving $Z$ exchange is four times smaller than was the case for the old luminosity detectors. This also reduces the size of the errors related to this contribution. The error in the hadronic part of the photon vacuum polarization in the $t$-channel is $0.05 \%$. The total theoretical error is estimated to be $0.25 \%$.
\end{abstract}

CERN-TH.6760/92

December 1992

\footnotetext{
*Present address: DESY, Notkestrasse 85, D-2000 Hamburg 52, Germany
} 
LEP has proved to be an excellent tool for precise tests of the Standard Model [1]. The precision of many of these tests hinges on the precision of the luminosity measurements. Up to now the level of accuracy reached by the LEP experiments in measuring the luminosity has been at most 0.45\% [2]. In September 1992, however, a new luminosity detector, a silicon-tungsten calorimeter (SICAL), has been installed in the ALEPH experiment [3], and a similar one will be mounted in the OPAL detector in 1993 [4]. These calorimeters are expected to bring down the experimental errors in determining the luminosity to roughly $0.1 \%$.

At LEP the luminosity is obtained by dividing the number of observed events in the luminosity detector by the predicted theoretical cross-section, taking into account the experimental efficiency. The precision of the theoretical prediction is therefore of the same importance as the precision of the measurements.

A very big effort has already been made to reduce the error of the theoretical prediction for the luminosity cross-section [5] - [11]. At present the LEP experiments use the Monte Carlo (MC) generator BHLUMI [9] to calculate the dominant photonic t-channel contribution. This generator contains $\mathrm{O}(\alpha)$ corrections and exponentiated multiphoton generation. It has a precision of $0.25 \%$ for the photonic t-channel contribution, as determined with the help of the benchmarks LUMLOG [8] and OLDBIS [6], both having a technical precision of $0.02 \%$. In our previous paper [11] we suggested to use the MC BABAMC [5] to calculate the $Z$-exchange contribution to the luminosity measurements at LEP and showed that the overall error on the luminosity calculation was below $0.3 \%$. Up to now the precision of the above-mentioned programs was tested in the angular region between $3.3^{\circ}$ and $6.3^{\circ}$. The new silicon-tungsten calorimeters, however, will be located at smaller angles. The purpose of this work is therefore the reanalysis of the magnitude of the various terms contributing to the luminosity calculation in this new angular region, and the verification of the precision with which they are calculated.

In this paper we will use the acceptance of the ALEPH SICAL detector. This acceptance is asymmetric, so as to reduce the sensitivity of the luminosity measurements to the interaction vertex position. The 'narrow' angular region ranges from $1.61^{\circ}$ to $2.8^{\circ}$ and the 'wide' one from $1.5^{\circ}$ to $3.15^{\circ}$. This defines three different cross-sections for our studies: 'narrow-narrow' (NN), 'wide-wide' (WW), and 'narrow-wide' (NW). The latter is the one that is actually measured in the experiment.

The following programs will be used in our analysis:

- OLDBIS: an $\mathrm{O}(\alpha)$ t-channel photon-exchange benchmark MC [6].

- BABAMC: a full $\mathrm{O}(\alpha)$ Bhabha scattering MC program containing Z-exchange terms [5].

- ALIBABA: a semi-analytical program, containing Z-exchange contributions, that calculates full $\mathrm{O}(\alpha)$ corrections and in addition resummation effects and 
higher-order multiphoton QED corrections in the leading-log approximation [7]. For our purposes we have used an unreleased luminosity version of this program.

- LUMLOG: a MC program calculating exponentiated leading-log initial-state corrections up to $\mathrm{O}\left(\alpha^{3}\right)$ for the $t$-channel photon exchange [8].

- BHLUMI: an $\mathrm{O}(\alpha)$ multiphoton MC generator with exclusive exponentiation of the Yennie-Frautschi-Suura type [9]. The contribution related to the interference between $t$-channel photon exchange and $s$-channel $Z$ exchange is included in the Born approximation.

In Table 1 the most important contributions to the Born cross-section for the NN acceptance are presented. It should be noted that at lowest-order level the NW cross-section is equal to the NN one. The matrix elements appearing in the various Born contributions are indicated by $G_{C}$, where $G$ stands for the exchanged gauge boson $(Z$ or $\gamma$ ) and $c$ for the channel ( $s$ or $t$ ). It should be noted that the $Z$ mass $M_{Z}=91.161 \mathrm{GeV}$ and the $Z$ width $\Gamma_{Z}$ are treated as input parameters in the various computer programs used throughout this paper. In order to calculate $\sin ^{2} \theta_{W}$, we used $M_{H}=100 \mathrm{GeV}$ and $m_{t}=130 \mathrm{GeV}$.

As a first step we investigate the size and reliability of the various corrections to the dominant $t$-channel photon-exchange contribution, as calculated by the MC programs now used in the LEP analyses. In the first part of Table 2 the QED $\mathrm{O}(\alpha)$ corrections of OLDBIS, BABAMC and ALIBABA are compared, for Bhabha scattering at $\sqrt{s}=$ $M_{Z}$, without cutting on the photon energy. To make the comparison with ALIBABA meaningful, only electrons and positrons were accepted within the above specified

Table 1: The most important contributions to the lowest-order Bhabha cross-section in the NN angular region. The other possible contributions are smaller than $0.001 \%$.

\begin{tabular}{|c|c|c|c|c|c|}
\hline \hline $\begin{array}{c}\sqrt{s} \\
(\mathrm{GeV})\end{array}$ & $\begin{array}{c}\text { NN Born } \\
(\mathrm{nb})\end{array}$ & $\begin{array}{c}\gamma_{t}-\gamma_{t} \\
(\% \text { Born })\end{array}$ & $\begin{array}{c}Z_{s}-\gamma_{t} \\
(\% \text { Born })\end{array}$ & $\begin{array}{c}\gamma_{s}-\gamma_{t} \\
(\% \text { Born })\end{array}$ & $\begin{array}{c}Z_{s^{-}} Z_{s} \\
(\% \text { Born })\end{array}$ \\
\hline \hline 89.661 & 110.07 & +99.816 & +0.216 & -0.033 & +0.000 \\
90.036 & 109.17 & +99.807 & +0.225 & -0.033 & +0.001 \\
90.411 & 108.25 & +99.824 & +0.207 & -0.033 & +0.001 \\
90.786 & 107.28 & +99.898 & +0.134 & -0.033 & +0.001 \\
91.161 & 106.25 & 100.031 & +0.000 & -0.033 & +0.001 \\
91.536 & 105.24 & 100.168 & -0.137 & -0.033 & +0.001 \\
91.911 & 104.30 & 100.247 & -0.215 & -0.033 & +0.001 \\
92.286 & 103.43 & 100.269 & -0.237 & -0.033 & +0.001 \\
92.661 & 102.60 & 100.263 & -0.231 & -0.033 & +0.000 \\
\hline \hline
\end{tabular}


Table 2: QED corrections to the $t$-channel photon-exchange cross-section at $\sqrt{s}=$ $M_{Z}$, calculated by different programs and presented as fractions (\%) of the full Born cross-sections of the NN (106.250 nb) and WW (141.396 nb) angular regions. The symbol $\Theta_{g e n}$ designates the $\mathrm{MC}$ generation region (see text).

\begin{tabular}{|c|c|c|c|}
\hline \multicolumn{4}{|c|}{ QED $\mathrm{O}(\alpha)$ corrections (\% of Born) } \\
\hline & NW & WW & $\mathrm{NN}$ \\
\hline OLDBIS & $-4-4.66 \pm 0.02$ & $-6.40 \pm 0.02$ & $-8.30 \pm 0.02$ \\
\hline BABAMC & $-4.73 \pm 0.01$ & $-6.44 \pm 0.01$ & $-8.33 \pm 0.01$ \\
\hline ALIBABA & $-4.83 \pm 0.16$ & $-6.31 \pm 0.15$ & $-8.24 \pm 0.16$ \\
\hline \multicolumn{4}{|c|}{ Leading-log fraction $(\%)$ of QED $\mathrm{O}(\alpha)$ corrections } \\
\hline$t_{\min }$ & 86 & 85 & 87 \\
\hline$t_{\max }$ & 92 & 93 & 93 \\
\hline \multicolumn{4}{|c|}{ Leading-log $\mathrm{O}\left(\alpha^{2}\right)$ corrections (\% of Born) } \\
\hline LUMLOG $t_{\min }$ & $+0.332 \pm 0.008$ & $+0.578 \pm 0.009$ & $+0.696 \pm 0.010$ \\
\hline ALIBABA $t_{\min }$ & $+0.331 \pm 0.001$ & $+0.583 \pm 0.001$ & $+0.695 \pm 0.001$ \\
\hline LUMLOG $t_{\max }$ & $+0.381 \pm 0.008$ & $+0.694 \pm 0.011$ & $+0.798 \pm 0.012$ \\
\hline ALIBABA $t_{\max }$ & $+0.379 \pm 0.001$ & $+0.701 \pm 0.001$ & $+0.797 \pm 0.001$ \\
\hline \multicolumn{4}{|c|}{ Higher-order leading-log corrections (\% of Born) } \\
\hline LUMLOG $t_{\min }$ & $-0.011 \pm 0.006$ & $-0.034 \pm 0.007$ & $-0.046 \pm 0.008$ \\
\hline ALIBABA $t_{\min }$ & $+0.042 \pm 0.001$ & $+0.022 \pm 0.001$ & $+0.024 \pm 0.001$ \\
\hline $\mathrm{ALIBABA}^{*} t_{\min }$ & $-0.015 \pm 0.001$ & $-0.043 \pm 0.001$ & $-0.054 \pm 0.001$ \\
\hline LUMLOG $t_{\max }$ & $-0.013 \pm 0.007$ & $-0.043 \pm 0.008$ & $-0.055 \pm 0.009$ \\
\hline ALIBABA $t_{\max }$ & $+0.051 \pm 0.001$ & $+0.034 \pm 0.001$ & $+0.027 \pm 0.001$ \\
\hline $\mathrm{ALIBABA}^{*} t_{\max }$ & $-0.020 \pm 0.001$ & $-0.058 \pm 0.001$ & $-0.066 \pm 0.001$ \\
\hline \multicolumn{4}{|c|}{ BHLUMI-OLDBIS-LUMLOG for a calorimetric trigger (\% of Born) } \\
\hline No $\Theta_{g e n}$ cut & $+0.094 \pm 0.037$ & $+0.200 \pm 0.045$ & $+0.208 \pm 0.054$ \\
\hline With $\Theta_{g e n}$ cut & $-0.030 \pm 0.037$ & $+0.079 \pm 0.045$ & $+0.112 \pm 0.053$ \\
\hline
\end{tabular}

angular regions. In order to get rid of the photon vacuum polarization and the $Z_{s^{-}}$ $\gamma_{t}$ interference, the weak corrections and the $Z$-exchange contributions were switched off in BABAMC, whereas in ALIBABA the weak corrections were switched off and only the $t$-channel contributions were considered. As we can see in Table 2 ALIBABA agrees with the other programs within its statistical error, related to the precision of the 5-dimensional hard photon Monte Carlo integration. The agreement between BABAMC and OLDBIS is better than $0.1 \%$ of the Born cross-section. The observed difference is related to the precision of $\mathrm{BABAMC}$ and does not invalidate the $0.02 \%$ technical precision of OLDBIS.

In order to study the quality of the leading- $\log (\mathrm{LL})$ approximation, the $\mathrm{O}(\alpha) \mathrm{LL}$ 
cross-section was calculated with ALIBABA and LUMLOG. Note that the statistical error in the ALIBABA LL calculation is reduced to well below $0.01 \%$ as a result of the fact that only a 2-dimensional integration has to be performed. The corresponding results were found to agree within the $0.01 \%$ statistical error of LUMLOG. In these LL calculations we required that both the $e^{+}$and $e^{-}$energies be above $1 \mathrm{GeV}$, so as to assure the reliability of the leading-log approximation, which is based on the smallness of the mass of the electrons and positrons as compared with their energies. Moreover, we made sure that the LL cross-sections only contained pure LL terms, i.e. terms of the form $(\alpha L / \pi)^{n}$, with $L=\log \left(Q^{2} / m_{e}^{2}\right)$ and $Q^{2} \gg m_{e}^{2}$. In the second part of Table 2 the LL fraction of the $\mathrm{O}(\alpha)$ QED corrections is given for different LL scale choices $Q^{2}$. In order to compare the results of the two programs and check their precision, we have chosen the scales $Q^{2}=-t_{\min }$ and $Q^{2}=-t_{\text {max }}$, corresponding to the $t$ values at the minimum and maximum angles of the considered angular acceptance. For the NW cross-sections, however, these scale choices are based on the NN angular acceptance.

In the third part of Table 2 the values for the multiphoton LL $\mathrm{O}\left(\alpha^{2}\right)$ corrections, as calculated by LUMLOG and ALIBABA, are given for different LL scale choices. The size of these corrections ranges from $0.3 \%$ to $0.8 \%$ of the corresponding full Born cross-sections. This makes the implementation of these terms indispensable. Once again LUMLOG and ALIBABA were found to agree within the $0.01 \%$ statistical error.

From the above-discussed $\mathrm{O}(\alpha)$ and $\mathrm{O}\left(\alpha^{2}\right)$ leading-log corrections, it can be inferred that the corresponding sub-leading $\mathrm{O}\left(\alpha^{2}\right)$ corrections could amount to as much as $0.1 \%$, in agreement with the value stated in [9].

We have explicitly checked that the LL $\mathrm{O}\left(\alpha^{2}\right)$ formulae in LUMLOG and ALIBABA were identical. For still higher-order corrections, differences appear. We will compare below the higher-order LL $\mathrm{O}\left(\alpha^{3}\right)$ exponentiated corrections in LUMLOG with the $\mathrm{O}\left(\alpha^{2}\right)$ exponentiated corrections in ALIBABA. In the fourth part of Table 2 these higher-order multiphoton LL corrections are given for different LL scale choices. They can amount to a non-negligible contribution of up to $0.05 \%$ of the full Born cross-section, depending on the angular region. The observed differences between LUMLOG and ALIBABA are caused by the difference in the way the leading-log structure functions are exponentiated, per initial-state particle (LUMLOG) or for the initial state as a whole (ALIBABA), and by the absence of $\mathrm{O}\left(\alpha^{3}\right)$ terms in the structure functions used in ALIBABA. After having included the dominant $\mathrm{O}\left(\alpha^{3}\right) \mathrm{LL}$ contributions in ALIBABA (see ALIBABA* entries), the two programs were found to agree at the $0.01 \%$ level.

Based on the above comparisons we conclude that the technical precision of $0.02 \%$ of LUMLOG determined in [8] still holds for the small-angle regions under investigation. Hence, the technical precision of the $\mathrm{O}(\alpha)$ QED corrections combined with the higher-order leading-log corrections (OLDBIS+LUMLOG) is $0.03 \%$ in this angular region.

Up to now only those corrections owing to multiphoton radiation have been dis- 
cussed. A remaining question is whether multiple photon bremsstrahlung is the only relevant $\mathrm{O}\left(\alpha^{2}\right)$ effect. To this end we studied the influence of the initial-state emission of electron-positron pairs going into the beam pipe. By means of an explicit LL calculation we investigated the potentially important contribution resulting from the emission of such a pair from one of the incoming particles via the transition of an electron to a photon and back again. In that case the lowest-order cross-section that enters the corresponding leading-log calculation is the same as the one used for the calculation of the leading bremsstrahlung contributions. Hence, intrinsically this contribution could be just as important as multiple bremsstrahlung. As a result of the splitting functions involved $\left(\Gamma_{\gamma e} \otimes \Gamma_{e \gamma}\right)$, the corresponding corrections exhibit a strong peaking for large energies of the emitted pair, so large corrections can be expected in that regime. For reasonable cuts on the energy of the final-state electron and positron, however, the corresponding contribution is negligible. Its size remains below $0.01 \%$ for an energy cut of roughly $15 \mathrm{GeV}$ on both the final-state electron and positron, taking into account the possibility that, in the actual luminosity measurement, an event where two electrons or two positrons satisfy the selection criteria is added to the luminosity sample. The case where the electron-positron pair is emitted from both initial-state particles is suppressed by at least an order of magnitude, as the lowest-order process entering the leading-log calculation involves two photons in the initial state and consequently has a suppressed peaking behaviour compared with the Bhabha scattering cross-section. Another potentially important contribution is obtained when the undetected electron-positron pair results from the conversion of a virtual photon emitted from the initial state. This contribution has to be combined with the corresponding electron-loop $\mathrm{O}\left(\alpha^{2}\right)$ corrections to Bhabha scattering to give contributions proportional to $(\alpha L / \pi)^{2}$. With the help of LUMLOG we have determined the corresponding corrections to lie between $-0.02 \%$ and $-0.04 \%$, depending on the angular region. The smallest corrections were found for the NW acceptance, as the size of this type of pair production is linked to the size of the LL $\mathrm{O}(\alpha)$ corrections. It should be noted that the above-discussed pair-production corrections are absent in the generators BABAMC and BHLUMI, which are used in the data analysis.

Until now we applied the acceptance cuts only to electrons and positrons, in order to compare results with the ALIBABA program. The majority of LEP detectors, however, does not distinguish these particles from photons. In order to study the BHLUMI precision for the new luminosity detectors, we therefore compare in the last part of Table 2 the BHLUMI results (see [9]) with the OLDBIS+LUMLOG results for a "calorimetric trigger". This trigger requires the energies of electrons, positrons, and photons, summed inside the angular regions defined above, to be larger than $0.25 \sqrt{s}$ in each detector. In addition the sum of energies in the two detectors should be larger than $0.6 \sqrt{s}$. All three programs were run for their default parameter and scale choice settings, and in both BHLUMI and OLDBIS the Monte Carlo events were generated between $1^{\circ}$ and $10^{\circ}$. In OLDBIS this means that only events with $e^{+}$and $e^{-}$within this angular range are generated. In BHLUMI this is translated to momentum transfer limits via the lowest-order relation $t=s(1-\cos \Theta)$, so that 
events with $e^{+}$and $e^{-}$outside the generation region can be present. These events were removed in the entries "with $\Theta_{\text {gen }}$ cut" by additionally imposing the generated $e^{+}$and $e^{-}$to have angles between $1^{\circ}$ and $10^{\circ}$. The BHLUMI-OLDBIS-LUMLOG tests in Ref. [9] were performed without a $\Theta_{\text {gen }}$ cut in the angular region between $3.3^{\circ}$ and $6.3^{\circ}$. The differences found in that angular region were below $0.15 \%$. From Table 2 we see that these differences can be larger for the angular regions covered in this paper. In the presence of the $\Theta_{g e n}$ cut, however, they drop below $0.15 \%$. Hence, we conclude that we can assign the same errors to BHLUMI as stated in Ref. [9].

The non-QED corrections to the $t$-channel photon-exchange contribution consist almost exclusively of the photon vacuum polarization. As this constitutes an important contribution to the cross-section, we have studied this term explicitly. In BABAMC it is implemented by adding the $\mathrm{O}(\alpha)$ correction $2 \mathrm{Re} \Pi=4.01 \%$ for the NW acceptance. This would correspond to a resummed value of roughly $4.13 \%$, which was found to agree at the $0.01 \%$ level with the ALIBABA result. Similar agreement was found for the other angular regions. In order to compare it with the vacuum polarization result in BHLUMI, the above resummed value has to be corrected for the $-4.22 \%$ exponentiated $\mathrm{O}(\alpha)$ correction of BHLUMI, leading to an overall vacuum polarization contribution of $3.96 \%$. Similar corrections were made for the symmetric acceptances, resulting in a mean agreement at the $0.02 \%$ level with the actual contributions in BHLUMI. We estimate the intrinsic systematic error in the calculation of the vacuum polarization to be $0.05 \%$, as the hadronic loops contribute $25-30 \%$ of the total $4 \%$ correction and have an error, resulting from the dispersion integral calculation, which is $4.5 \%$ of this contribution [12].

As can be read off in Table 1 the contributions originating from the interference between photon exchange in the $t$-channel and $Z$ exchange in the $s$-channel give rise to a non-negligible energy dependence, with a maximum value of more than $0.2 \%$ of the full Born cross-section. In [11] it was observed that the corresponding $O(\alpha)$ QED corrections can reach $50 \%$ of the peak value at lowest order in the angular region between $3.3^{\circ}$ and $6.3^{\circ}$. This effect is predominantly the result of the $Z$ resonance and is hence expected to take comparable proportions in the angular regions under investigation. Consequently these $\mathrm{O}(\alpha)$ QED corrections are indispensable for reducing the theoretical uncertainty below the expected experimental one. Confirmation of all this can be found in Table 3 , for the NW angular region. In this table the $\mathrm{O}(\alpha)$ corrections to all $Z$-exchange contributions are given, rather than just the corrections to the $Z_{s}-\gamma_{t}$ interference. This is done in order to facilitate the isolation of these terms in BABAMC. The $\mathrm{O}(\alpha)$ ALIBABA entries in this table involve values for $\sin ^{2} \theta_{W}(=0.2273)$ and $\Gamma_{Z}(=2.3098 \mathrm{GeV})$ that are set equal to the ones used in BABAMC, so as to compensate for the improvements, over the last couple of years, in the calculation of these quantities. To avoid confusion we will indicate the lowest-order contribution corresponding to the $Z$-boson exchange by $Z$-Born. From the comparison between the BABAMC and $\mathrm{O}(\alpha)$ ALIBABA results, which have an error of about $0.001 \%$ (see [11]), we infer a $0.03 \%$ technical precision for the $Z_{s}-\gamma_{t}$ interference contribution of BABAMC in the NW angular region. 
Table 3: $\mathrm{O}(\alpha)$ corrections to the $Z$-boson exchange contribution calculated with BABAMC, compared with the results of $\mathrm{O}(\alpha)$ ALIBABA. All cross-sections are presented as fractions (\%) of the full Born cross-section.

\begin{tabular}{|c|c|c|c|c|c|c|}
\hline \hline$\sqrt{s}$ & \multicolumn{3}{|c|}{ QED O $(\alpha)$} & \multicolumn{3}{c|}{ Remaining $\mathrm{O}(\alpha)$} \\
\hline $\mathrm{GeV}$ & BABAMC & ALIBABA & Difference & BABAMC & ALIBABA & Difference \\
\hline \hline 89.661 & -0.059 & -0.058 & -0.001 & +0.013 & +0.012 & +0.001 \\
90.036 & -0.059 & -0.059 & +0.000 & +0.002 & +0.009 & -0.007 \\
90.411 & -0.042 & -0.048 & +0.006 & -0.015 & +0.004 & -0.019 \\
90.786 & -0.002 & -0.012 & +0.010 & -0.021 & +0.000 & -0.021 \\
91.161 & +0.054 & +0.049 & +0.005 & -0.012 & +0.003 & -0.015 \\
91.536 & +0.100 & +0.101 & -0.001 & -0.008 & +0.010 & -0.018 \\
91.911 & +0.129 & +0.117 & +0.012 & -0.013 & +0.010 & -0.023 \\
92.286 & +0.121 & +0.109 & +0.012 & -0.020 & +0.006 & -0.026 \\
92.661 & +0.088 & +0.092 & -0.004 & -0.022 & +0.001 & -0.023 \\
\hline \hline
\end{tabular}

Table 4: $\mathrm{O}(\alpha)$ corrections and corrected results for the $Z$-boson exchange contributions taken from BABAMC using a $Z$ width of $2.487 \mathrm{GeV}$ and the differences to the corresponding results calculated with a $Z$ width of $2.3098 \mathrm{GeV}$ (default $Z$ width in BABAMC, see text and [11] for more details). All cross-sections are presented as fractions (\%) of the Born cross-section given in Table 1.

\begin{tabular}{|c|c|c|c|c|c|c|}
\hline \hline$\sqrt{s}$ & \multicolumn{2}{|c|}{$Z$-Born $+\mathrm{O}(\alpha)$ corr. } & \multicolumn{2}{|c|}{ QED $\mathrm{O}(\alpha)$} & \multicolumn{2}{c|}{ Remaining $\mathrm{O}(\alpha)$} \\
\hline $\mathrm{GeV}$ & 2.487 & Difference & 2.487 & Difference & 2.487 & Difference \\
\hline \hline 89.661 & +0.176 & +0.006 & -0.056 & +0.003 & +0.028 & +0.015 \\
90.036 & +0.172 & +0.004 & -0.053 & +0.006 & +0.017 & +0.015 \\
90.411 & +0.156 & +0.005 & -0.037 & +0.005 & +0.006 & +0.021 \\
90.786 & +0.119 & +0.007 & +0.016 & +0.018 & -0.014 & +0.007 \\
91.161 & +0.036 & -0.007 & +0.049 & -0.005 & -0.014 & -0.002 \\
91.536 & -0.048 & -0.004 & +0.089 & -0.014 & -0.018 & -0.010 \\
91.911 & -0.105 & -0.007 & +0.120 & -0.009 & -0.032 & -0.019 \\
92.286 & -0.141 & -0.006 & +0.103 & -0.018 & -0.025 & -0.005 \\
92.661 & -0.156 & +0.008 & +0.082 & -0.006 & -0.021 & +0.001 \\
\hline \hline
\end{tabular}

If, as in [11], the input value of the $Z$ width is changed from $2.3098 \mathrm{GeV}$, as calculated in the Born approximation with BABAMC, to $2.487 \mathrm{GeV}$, as measured by the LEP collaborations [1], a negligible effect is observed (see Table 4). 
Table 5: Higher-order corrections to the Z-boson exchange contributions determined relative to the $\mathrm{O}(\alpha)$ corrected results based on $\Gamma_{Z}=2.3098 \mathrm{GeV}$ (diff.1) or $\Gamma_{Z}=2.487 \mathrm{GeV}$ (diff.2). All cross-sections are taken from the corresponding ALIBABA versions and are presented as fractions (\%) of the Born cross-section given in Table 1.

\begin{tabular}{|c|c|c|c|c|c|c|c|}
\hline \hline$\sqrt{s}$ & \multicolumn{2}{|c|}{ Z-Born } & \multicolumn{2}{|c|}{ Z-Born $+\mathrm{O}(\alpha)$} & \multicolumn{3}{c|}{ Z-Born $+\mathrm{O}(\alpha)+$ h.o. } \\
\hline & 2.3098 & 2.487 & 2.3098 & 2.487 & & diff.1 & diff.2 \\
\hline \hline 89.661 & +0.216 & +0.204 & +0.170 & +0.172 & +0.172 & +0.002 & +0.000 \\
90.036 & +0.226 & +0.209 & +0.176 & +0.180 & +0.177 & +0.001 & -0.003 \\
90.411 & +0.208 & +0.187 & +0.164 & +0.169 & +0.164 & +0.000 & -0.005 \\
90.786 & +0.135 & +0.118 & +0.124 & +0.127 & +0.117 & -0.007 & -0.010 \\
91.161 & +0.001 & +0.001 & +0.054 & +0.050 & +0.036 & -0.018 & -0.014 \\
91.536 & -0.136 & -0.119 & -0.026 & -0.036 & -0.050 & -0.024 & -0.014 \\
91.911 & -0.214 & -0.193 & -0.087 & -0.096 & -0.110 & -0.023 & -0.014 \\
92.286 & -0.236 & -0.218 & -0.122 & -0.127 & -0.136 & -0.014 & -0.009 \\
92.661 & -0.231 & -0.218 & -0.137 & -0.140 & -0.145 & -0.008 & -0.005 \\
\hline \hline
\end{tabular}

Table 6: Summary of theoretical errors on the luminosity calculation in different angular regions.

\begin{tabular}{|l|c|c|}
\hline \hline \multicolumn{1}{|c|}{ Contribution } & NW & $3.3^{\circ}-6.3^{\circ}$ \\
\hline \hline$(1) \mathrm{O}\left(\alpha^{2}\right)$ LL BHLUMI & 0.15 & 0.15 \\
$(2) \mathrm{O}\left(\alpha^{2}\right)$ SL BHLUMI & 0.09 & 0.09 \\
$(3) Z$ exchange $\mathrm{O}(\alpha)$ BABAMC & 0.03 & 0.03 \\
$(4) Z$ exchange $\mathrm{O}\left(\alpha^{2}\right)$ LL BABAMC & 0.015 & 0.06 \\
$(5) Z$ exchange $\mathrm{O}\left(\alpha^{2}\right)$ SL BABAMC & 0.015 & 0.06 \\
$(6)$ Vacuum polarization & 0.05 & 0.08 \\
\hline Total theoretical error & 0.25 & 0.28 \\
\hline \hline
\end{tabular}

The higher-order effects, comprising resummation and leading-log QED effects, are investigated using the unadapted ALIBABA program. The corresponding results can be found in Table 5, and turn out to be negligibly small. As in [11] we determine from Table 5 the error in BABAMC related to missing $Z$-exchange LL O $\left(\alpha^{2}\right)$ corrections to be $0.015 \%$ and conservatively estimate the error related to missing sub-leading (SL) $\mathrm{O}\left(\alpha^{2}\right)$ corrections to be also $0.015 \%$.

In Table 6 we give a summary of the theoretical errors for the NW angular region 
compared with those in the angular region between $3.3^{\circ}$ and $6.3^{\circ}$ taken from our previous paper [11]. The first two quoted errors describe the BHLUMI precision and are taken from Ref. [9]. The remaining BHLUMI errors quoted in Ref. [9] are below $0.02 \%$ and are not presented in Table 6 . Since the leading- $\log \mathrm{O}\left(\alpha^{2}\right)$ corrections (LL) and the sub-leading $\mathrm{O}\left(\alpha^{2}\right)$ corrections (SL) can be correlated, we have added the errors of (1) and (2), as well as (4) and (5), linearly. The other errors were added in quadrature. We observe that for the angular region studied in this paper the error related to the $Z$-exchange contribution is substantially smaller and that also the error related to the photon vacuum polarization is smaller. Both these errors are well below the expected experimental precision of the new luminosity detectors. In order to reach a total theoretical precision of $0.1 \%$, a BHLUMI precision of $0.08 \%$ would be required. In view of this a calculation of the sub-leading $\mathrm{O}\left(\alpha^{2}\right)$ terms might have to be considered.

We would like to thank S. Jadach, E. Richter-Wạs, B.F.L. Ward and Z. Wạs for many stimulating discussions on this work. 


\section{References}

[1] The LEP Collaborations, Phys. Lett. B276 (1992) 247; L. Rolandi, CERNPPE/92-175, to appear in the Proc. of the XXVI ICHEP 1992, Dallas, USA; R. Tanaka, Ecole Polytechnique preprint X-LPNHE/92-3, to appear in the Proc. of the XXVI ICHEP 1992, Dallas, USA.

[2] H. Meinhard, "Precise Measurement of the Luminosity at LEP", to appear in the Proc. of the XXVI ICHEP 1992, Dallas, USA.

[3] J. Rander, ALEPH note 90-008, ALEPH, CERN; J. Rander and M. Martinez, ALEPH note 90-143, ALEPH, CERN.

[4] The OPAL Collaboration, "Proposal for Upgrading the OPAL Luminosity Detector", CERN/LEPC 91-8, LEPC/M-100.

[5] M. Böhm, A. Denner and W. Hollik, Nucl. Phys. B304 (1988) 687; F.A. Berends, R. Kleiss and W. Hollik, Nucl. Phys. B304 (1988) 712.

[6] S. Jadach, E. Richter-Wạs, Z. Wạs and B.F.L. Ward, Phys. Lett. B253 (1991) 469.

[7] W. Beenakker, F.A. Berends and S.C. van der Marck, Nucl. Phys. B349 (1991) 323 and B355 (1991) 281.

[8] S. Jadach, E. Richter-Wa̧s, Z. Wạs and B.F.L. Ward, Phys. Lett B260 (1991) 438.

[9] S. Jadach, E. Richter-Wąs, Z. Wa̧s and B.F.L. Ward, Phys. Lett. B268 (1991) 253; Comput. Phys. Commun. 70 (1992) 305; S. Jadach and B.F.L. Ward, Phys. Rev. D40 (1989) 3582.

[10] M. Cacciari, A. Deandrea, G. Montagna, O. Nicrosini and L. Trentadue, Phys. Lett. B271 (1991) 431.

[11] W. Beenakker and B. Pietrzyk, Phys. Lett. B296 (1992) 241.

[12] H. Burkhardt, F. Jegerlehner, G. Penso and C. Verzegnassi, Z. Phys. C43 (1989) 497. 